OPEN ACCESS

Edited by:

Xuming Deng,

Jilin University, China

Reviewed by:

Wenkai Ren,

South China Agricultural University,

China

Aart Lammers,

Wageningen University \& Research,

Netherlands

Ning $L i$,

Shandong Agricultural University,

China

*Correspondence:

Yantao Wu

ytwu@yzu.edu.cn

tThese authors have contributed equally to this work

Specialty section:

This article was submitted to

Food Microbiology,

a section of the journal

Frontiers in Microbiology

Received: 11 March 2020

Accepted: 06 July 2020

Published: 22 July 2020

Citation:

Guo M, Li M, Zhang C, Zhang X and Wu Y (2020) Dietary Administration of the Bacillus subtilis

Enhances Immune Responses and Disease Resistance in Chickens.

Front. Microbiol. 11:1768.

doi: 10.3389/fmich.2020.01768

\section{Dietary Administration of the Bacillus subtilis Enhances Immune Responses and Disease Resistance in Chickens}

\author{
Mengjiao Guo ${ }^{1 t}$, Mingtao $\mathrm{Li}^{1+}$, Chengcheng Zhang ${ }^{1}$, Xiaorong Zhang ${ }^{1}$ and Yantao Wu ${ }^{1,2 *}$ \\ 1 Jiangsu Co-innovation Center for Prevention and Control of Important Animal Infectious Diseases and Zoonoses, College \\ of Veterinary Medicine, Yangzhou University, Yangzhou, China, ${ }^{2}$ Joint International Research Laboratory of Agriculture \& \\ Agri-Product Safety (JIRLAAPS), Yangzhou University, Yangzhou, China
}

Bacillus subtilis (B. subtilis) has a variety of proposed beneficial effects for chickens, including growth promotion and disease prevention. In this study, chickens were fed a diet containing $B$. subtilis for 21 days and growth performance, intestinal morphology, intestinal microbiota, immune responses, and disease resistance were investigated. After 21 days of feeding, chickens fed a diet containing B. subtilis had higher body weights. The concentrations of serum immunoglobulins IgA and IgM were significantly increased by $B$. subtilis in the diet. Moreover, chickens fed with $B$. subtilis had greater villus height $(\mathrm{VH})$, shallower crypt depth $(\mathrm{CD})$, and a higher $\mathrm{VH} / \mathrm{CD}$ ratio in the jejunum than chickens fed a standard control diet. Diet with $B$. subtilis can balance intestinal microbiota, facilitate an increase in beneficial bacteria, and inhibit the pathogenic bacteria after 21 days of feeding. After an Escherichia coli (E. coli) challenge, the survival rate of chickens fed with $B$. subtilis was $66.67 \%$, which was significantly higher than the controls. The $E$. coli contents in spleens and lungs from chickens fed a diet with $B$. subtilis were lower than those in controls. In addition, $B$. subtilis can trigger the toll-like receptor 4 and cause induction of proinflammatory cytokine (II1 $\beta$, II6, and I/8) production to develop innate immune responses in chickens. In conclusion, diets containing $B$. subtilis can improve growth performance, serum immunoglobulin levels, the intestinal villus-crypt system, intestinal homeostasis, immune responses, and disease resistance against $E$. coli in chickens.

Keywords: Bacillus subtilis, growth performance, intestinal microbiota, immunity response, Escherichia coli

\section{INTRODUCTION}

Since 2006, growth-promoting antibiotics have been banned in Europe. Since 2020, all forms of growth-promoting antibiotics except traditional Chinese medicines have been forbidden to be used as feed additives in China. At present, with the increase in the number of laws and regulations concerning the use of antibiotics, and the difficulty of new drugs development, thus increasing the demand for growth promoter alternatives (Lupindu et al., 2015). Probiotics are live microorganisms that confer health benefits to the host to improve nutrient digestibility, improve gut health, enhance intestinal barrier integrity, and modulate immune responses (Fuller, 1989; Lee et al., 2010; 
Pagnini et al., 2010; Knap et al., 2011; Abdelqader et al., 2013). It has become a safe alternative that can partly replace antibiotics. Due to the strong resistance of spore to extreme conditions and long term storage, Bacillus spp., as probiotics, are recognized as the most suitable probiotics (Hong et al., 2005). Recent studies have confirmed that dietary inclusion of Bacillus spp. have beneficial effects in chickens (Al-Fataftah and Abdelqader, 2014; Lee et al., 2015; Whelan et al., 2019).

Among the wide varieties of bacterial species used as probiotics, Bacillus subtilis (B. subtilis) has been considered to be safe and effective for animals (EFSA, 2007). B. subtilis is well known for its capability to improve growth performance, compete with pathogens, balance intestinal microbiota, and improve disease resistance (Elshaghabee et al., 2017; Abudabos et al., 2019). Balanced intestinal microbiota benefit the host by competing for nutritional sources and adhesion sites of pathogens, promoting commensal proliferation, and improving the intestinal immune system ( $\mathrm{Ng}$ et al., 2009; Ma et al., 2018). In poultry, avian colibacillosis causes different syndromes in 3-12 week old broiler chickens that usually present a systemic infection with characteristic fibrinous lesions and septicemia (Schouler et al., 2012). At the outbreak period of avian colibacillosis, serious threats to poultry industry occurred. It was reported that dietary B. subtilis-based direct-fed microbials promoted growth performance and balanced the gastrointestinal microbiota by inhibiting the colonization of avian pathogenic Escherichia coli (E.coli) and Clostridium perfringens (Gebert, 2007; Lee S.H. et al., 2014). In addition, dietary direct-fed microbials alleviate clinical symptoms of ubiquitous poultry diseases, such as necrotic enteritis and Salmonellosis (Abudabos et al., 2019; Sokale et al., 2019).

Different presumable mechanisms for probiotic actions have been proposed and investigated, including inhibition and stimulation of host immunity. Innate immunity is the first-line of defense against the pathogens, and it has been demonstrated that dietary B. subtilis increases innate and acquired immune responses (Pagnini et al., 2010; Gadde et al., 2017). As expected, B. subtilis was shown to modulate host protective immune responses against bacterial infections (Rajput et al., 2014). However, the beneficial effects of $B$. subtilis are markedly straindependent as many properties vary as a function of strain (Larsen et al., 2014; Dowarah et al., 2018). In our previous study, B. subtilis BG5 and BYS2 strains were selected based on their beneficial effects in the gastrointestinal tract and inhibition of the growth of pathogenic bacteria (Guo et al., 2017). To gain better insight into the role of $B$. subtilis, the effects of dietary B. subtilis on growth performance, the intestinal villus-crypt system, intestinal microbiota, immune responses, and resistance to $E$. coli were investigated in chickens.

\section{MATERIALS AND METHODS}

\section{B. subtilis and Pathogen}

The probiotics, B. subtilis BG5 and BYS2, were isolated and characterized according to a previous study (Guo et al., 2017). A single colony was cultured in nutrient broth medium (Hopebio,
Qingdao, China) at $37^{\circ} \mathrm{C}$ for $12 \mathrm{~h}$. The probiotic microbe count was $10^{8} \mathrm{cfu} / \mathrm{mL}$.

The bacterial pathogen, E. coli (O1K1), was isolated from clinically infected ducks and grown in Luria-Bertani medium at $37^{\circ} \mathrm{C}$ for $10 \mathrm{~h}$. The bacterial suspension was prepared for the infection experiment in chickens at a concentration of $10^{7} \mathrm{cfu} / \mathrm{mL}$.

\section{Experimental Design}

One-day-old chickens (Ross 308) were purchased from a commercial hatchery and randomly allotted to two groups. Chickens of control group were fed with standard control diet (Table 1). Chickens of B. subtilis group were fed with $10^{6} \mathrm{cfu} / \mathrm{g}$ B. subtilis (mixture of BYS2 and BG5). Each group consisted of three replicates with 30 chickens per replicate. The chickens were weighed individually, and blood samples were collected through the wing vein at days 7,14 , and 21 . On days 14 and 21 , five chickens were randomly selected from each replicate and euthanized. Spleens were collected and stored at $-70^{\circ} \mathrm{C}$ for RNA extraction. On day 21, the chickens were challenged with E. coli. All experiments were carried out in accordance with the principles of the Basal Declaration and Recommendations of Committee on the Ethics of Animal Experiments of Yangzhou University. The protocol was approved by the Committee on the Ethics of Animal Experiments of Yangzhou University.

\section{Histomorphological Analysis}

On day 21, intestinal samples from the middle part of duodenum, jejunum, and ileum were collected and submerged in $4 \%$ paraformaldehyde solution. Intestinal samples were sliced using a microtome and then stained with hematoxylin and eosin (H\&E). Intestinal morphology was observed with a Leica microscope. Villus height was measured from the tip of the villus to the villus-crypt junction. Crypt depth was measured the depth of the invagination between adjacent villi. A total of 10 intact and well-oriented crypt-villus units were selected from each intestinal cross-section. The morphological measurements were

\begin{tabular}{lccc}
\hline \multicolumn{3}{c}{ TABLE 1 | Composition of the standard diet. } & \\
\hline Ingredients & $\begin{array}{c}\text { Percentage } \\
\mathbf{( \% )}\end{array}$ & $\begin{array}{c}\text { Nutrient } \\
\text { levels }\end{array}$ & $\begin{array}{c}\text { Content } \\
\mathbf{( \% )}\end{array}$ \\
\hline Corn & 66.50 & $\mathrm{ME} /(\mathrm{MJ} / \mathrm{kg})$ & 11.97 \\
Soybean meal & 27.00 & $\mathrm{CP}$ & 19.06 \\
Fish meal & 2.00 & Met & 0.47 \\
CaHPO 4 & 1.70 & Lys & 0.89 \\
Limestone & 1.10 & $\mathrm{Ca}$ & 0.98 \\
DL-methionien & 0.20 & $\mathrm{TO}$ & 0.71 \\
L-Lysine & 0.20 & & \\
NaCl & 0.30 & & \\
Premix & 1.00 & & \\
Total & 100 & & \\
\hline
\end{tabular}

aPremix provided the following each kilogram of diets:VA $10000 \mathrm{U}$; VB, $5 \mathrm{mg}$; $V B_{2} 10.0 \mathrm{mg} ; V_{6} 8.2 \mathrm{mg} ; \mathrm{VB}_{12} 40.0 \mathrm{mg} ; V_{3} 6000 \mathrm{U} ; \mathrm{VE} 50 \mathrm{U} ; \mathrm{VK}_{3} 3.0 \mathrm{mg}$, pantothenic acid $45.0 \mathrm{mg}$; biotin $2.0 \mathrm{mg}$; folic acid $1.0 \mathrm{mg}$; Cu $8.0 \mathrm{mg}$; Fe $80.0 \mathrm{mg}$; Mn 80.0 mg; Zn 90.0 mg; / 1.5 mg; Se 0.3 mg. 
made in $10-\mu \mathrm{m}$ increments with Image-Pro Plus software version 6 (Media Cybergenetics, United States) (Sen et al., 2012; AlFataftah and Abdelqader, 2014).

\section{$16 S$ rDNA Gene Sequencing and Analysis}

Cecal contents were collected for microbial analyses after 21 days of feeding. Total genomic DNA from samples was extracted using Hipure Soil DNA Kit (Magen, Guangzhou, China) according to the manufacturer's protocols. DNA concentration was monitored by Qubit 3.0 Fluorometer. The V3 and V4 hypervariable regions of prokaryotic $16 \mathrm{~S}$ rDNA were amplified using forward primer CCTACGGRRBGCASCAGKVRVGAAT and reverse primer GGACTACNVGGGTWTCTAATCC. DNA libraries $(10 \mathrm{nM})$ were multiplexed and loaded onto an Illumina MiSeq or NovaSeq instrument according to manufacturer's instructions (Illumina, San Diego, CA, United States). After quality filtering, the chimeric sequence was removed, and the final sequence was used for operational taxonomic unit (OTU) clustering using Vsearch clustering (1.9.6) with 97\% sequence similarity. The Ribosomal Database Program (RDP) classifier Bayesian algorithm of the OTU species taxonomy was then used to analyze representative sequences and under different species classification levels, statistical community compositions of each sample were performed. Based on the OTU analysis results and by using the method of random flattening sample sequences, the alpha diversity indices of ace, shannon, chao 1, and simpson were calculated by Qiime (1.9.1) software to reflect the species richness and diversity.

\section{Susceptibility of Chickens to E. coli}

All the chickens were intraperitoneal injections with $0.5 \mathrm{~mL}$ of bacterial suspension $\left(10^{7} \mathrm{cfu} / \mathrm{mL}\right)$. The unchallenged control received $0.5 \mathrm{~mL}$ saline. After infection for 1 and 3 days, $E$. coli counts were determined by analyzing the hearts, livers, spleens, lungs, and kidneys from five chickens per group. The collected tissue samples were mixed with PBS $(1 \mathrm{~mL} / \mathrm{g})$ and were ground into tissues homogenate. Then tissue homogenates were 10 -fold diluted with PBS, and plated onto Luria-Bertani agar to calculate the E. coli CFU. The survival rate of chickens was calculated at the end of the experiment.

\section{Effect of Dietary B. subtilis on Transcript Level of Immunity-Related Genes}

To measure the expression of immunity-related genes, RNA was extracted from spleen samples using TRIzon Reagent (CoWin Biosciences, Beijing, China). Reverse transcription was performed with $\mathrm{HiScript}^{R}$ II Q Select RT SuperMix for realtime polymerase chain reaction ( $\mathrm{PPCR}+$ gDNA wiper) (Vazyme, Nanjing, China). $\beta$-actin and immune-related gene primers for quantitative qPCR oligonucleotides are listed in Table 2. qPCR was performed with the Applied Biosystems 7500 Fast RealTime PCR System (Applied Biosystems, CA, United States). qPCR reactions were performed in a total volume of $20 \mu \mathrm{L}$ with the SYBR Green PCR kit (Transgen Biotech Co., Ltd., Beijing, China). qPCR reactions were run using a specific cycling protocol: $30 \mathrm{~s}$ at $94^{\circ} \mathrm{C} ; 40$ cycles of $5 \mathrm{~s}$ at $94^{\circ} \mathrm{C} ; 34 \mathrm{~s}$ at $60^{\circ} \mathrm{C}$;
TABLE 2 | Primers used in this study.

\begin{tabular}{|c|c|c|}
\hline Primer name & Sequence $\left(5^{\prime}-3^{\prime}\right)$ & GenBank number \\
\hline TIr4 F & AGTCTGAAATTGCTGAGCTCAAAT & AY064697 \\
\hline TIr4 R & GCGACGTTAAGCCATGGAAG & \\
\hline Mhc II- $\alpha \mathrm{F}$ & TGGGATCCTCCGTCCTGAAGCCGCAC & EF554707.1 \\
\hline Mhc II- $\alpha \mathrm{R}$ & GCGTCGACTCAGAGCAGCCCCGGTT & \\
\hline Myd88 F & TGATGCCTTCATCTGCTACTG & EF011109 \\
\hline Myd88 R & TCCCTCCGACACCTTCTTTCTA & \\
\hline$N F-\kappa B F$ & CAGCCCATCTATGACAACCG & NM-205129 \\
\hline$N F-\kappa B R$ & TCCCTGCGTCTCCTCTGTGA & \\
\hline Ifn- $\alpha \mathrm{F}$ & ATGCCACCTTCTCTCACGAC & EU367971 \\
\hline Ifn- $\alpha \mathrm{R}$ & AGGCGCTGTAATCGTTGTCT & \\
\hline$\| 1 \beta F$ & GTGAGGCTCAACATTGCGCTGTA & NM204524.1 \\
\hline$\| 1 \beta R$ & TGTCCAGGCGGTAGAAGATGAAG & \\
\hline $116 \mathrm{~F}$ & TCTGTTCGCCTTCAGACCTA & AJ309540 \\
\hline $116 \mathrm{R}$ & GACCACCTCATCGGGATTTAT & \\
\hline $1 / 8 \mathrm{~F}$ & ATGAACGGCAAGCTTGGAGCTG & DQ393272.2 \\
\hline $1 / 8 \mathrm{R}$ & TCCAAGCACACCTCTCTTCCATCC & \\
\hline$\beta$-actin $\mathrm{F}$ & GAGAAATTGTGCGTGACATCA & L08165.1 \\
\hline$\beta$-actin R & ССTGAACСTCTCATTGCCA & \\
\hline
\end{tabular}

and finally with a dissociation curve. The relative expressions of mRNA were calculated based on the $2^{-\Delta \Delta C t}$ method. Target gene mRNAs were normalized to $\beta$-actin of each sample. Each sample was run in triplicate. The results were expressed as the B. subtilis vs. the control group.

\section{Statistical Analysis}

The non-parametric Mann-Whitney $U$ test was conducted to examine significant differences between $B$. subtilis and control group using the SPSS computer software (SPSS Inc., Chicago, IL, United States). The survival rate of chickens was calculated by Kaplan-Meier method and the significant difference was analyzed by Log-rank test. A value of $P<0.05$ was considered significant.

\section{RESULTS}

\section{Growth Performance and Serum Immunoglobulin}

The chickens fed diet with B. subtilis had higher body weight than chickens fed standard control diet, especially at 21 days of feeding (Figure 1A). After 14 and 21 days of feeding, serum IgA of chickens fed with $B$. subtilis were significantly higher $(P<0.05)$ than that of controls (Figure 1B). However, no significant differences was observed in serum IgG between chickens fed with B. subtilis diets and the controls (Figure 1C). Chickens fed diets containing B. subtilis showed higher IgM levels than the controls at 21 days (Figure 1D).

\section{Small Intestinal Morphology}

To gain better insight into the effect of $B$. subtilis on intestinal morphology, the duodenum, jejunum, and ileum were collected for histomorphological analyses (Supplementary Figure 1). As shown in Table 3, chickens fed with B. subtilis had greater villus 

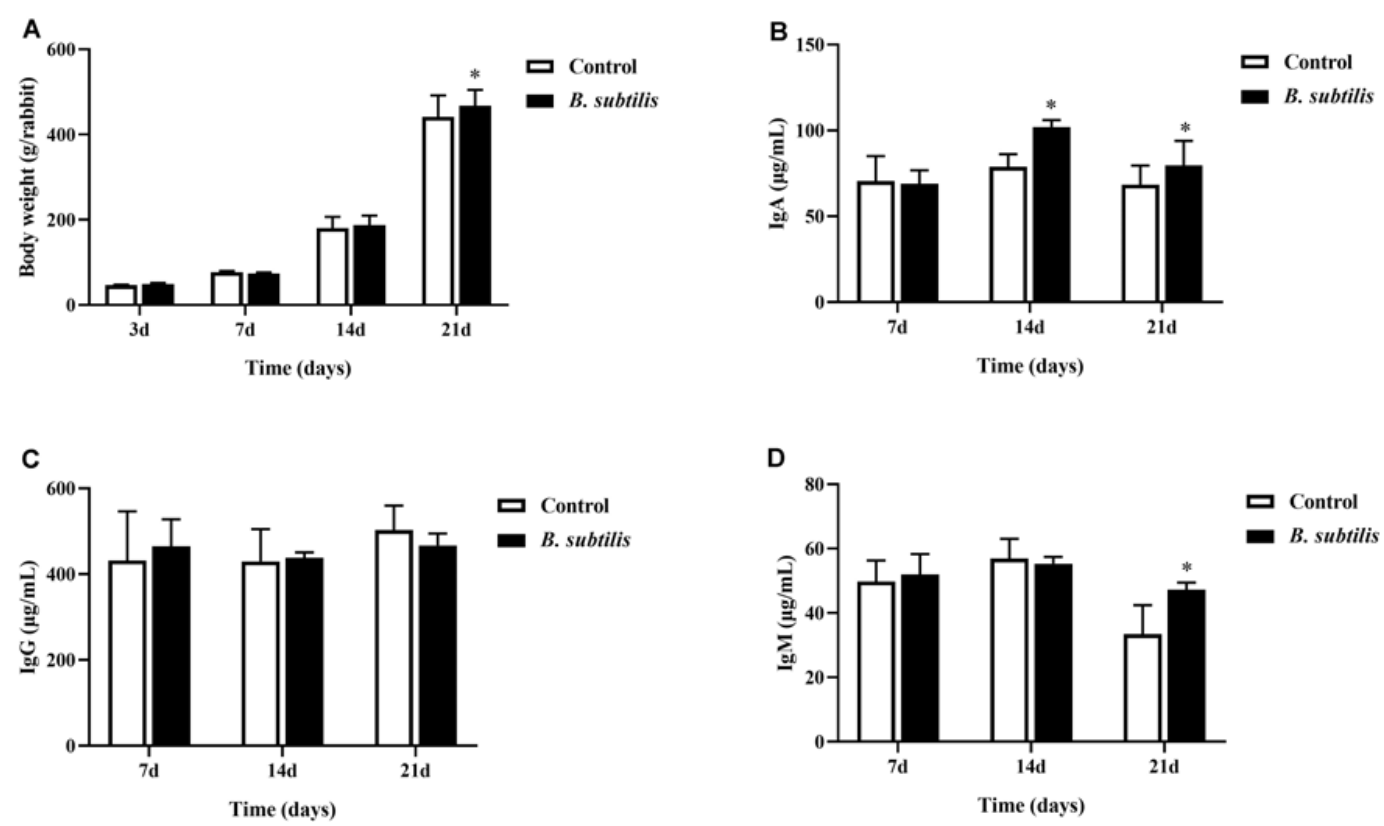

FIGURE 1 | Effects of dietary Bacillus subtilis on growth performance and serum immunoglobulin of chickens. (A) The body weights were measured at 3, 7, 14, and 21 days after feeding B. subtilis. The concentrations of $\operatorname{lgA}(\mathbf{B}), \lg G(\mathbf{C})$, and $\lg M(\mathbf{D})$ were measured at 7,14 , and 21 days after feeding B. subtilis. Bars were expressed as means \pm standard deviations $(n=5)$. ${ }^{*} P<0.05$.

heights $(\mathrm{VH})$ of jejunum $(P<0.05)$ and ileum at 21 days. The crypt depth $(\mathrm{CD})$ of the duodenum, jejunum $(P<0.05)$, and ileum were shallower in chickens fed with $B$. subtilis than those fed with the control diet. The villus height: crypt depth (VH/CD) ratio of the duodenum, jejunum, and ileum were higher $(P<0.05)$ in chickens fed with $B$. subtilis than the control.

\section{Taxonomic Composition of Intestinal Microbiota}

To determine how B. subtilis regulates intestinal microbiota, 16S rDNA sequences of cecal contents were analyzed at 21 days.

TABLE 3 | Effects of dietary Bacillus subtilis on intestinal villus-crypt system.

\begin{tabular}{lccc}
\hline & & Control & Bacillus subtilis \\
\hline Duodenum & & & \\
& & & \\
& Villus height $(\mu \mathrm{m})$ & $1507.67 \pm 202.30$ & $1579.00 \pm 54.69$ \\
& Crypt depth $(\mu \mathrm{m})$ & $194.00 \pm 8.54$ & $187.67 \pm 12.93$ \\
Jejunum & VH/CD & $7.77 \pm 1.25^{\mathrm{a}}$ & $8.41 \pm 0.51^{\mathrm{b}}$ \\
& & & \\
& Villus height $(\mu \mathrm{m})$ & $749.33 \pm 106.44^{\mathrm{a}}$ & $903.00 \pm 107.87^{\mathrm{b}}$ \\
& Crypt depth $(\mu \mathrm{m})$ & $210.08 \pm 33.99^{\mathrm{a}}$ & $141.33 \pm 17.80^{\mathrm{b}}$ \\
& VH/CD & $3.57 \pm 0.61^{\mathrm{a}}$ & $6.39 \pm 0.77^{\mathrm{b}}$ \\
& & & \\
& & & \\
& Villus height $(\mu \mathrm{m})$ & $606.17 \pm 71.77$ & $555.75 \pm 15.09$ \\
& Crypt depth $(\mu \mathrm{m})$ & $154.75 \pm 28.03$ & $119.56 \pm 15.87$ \\
& VH/CD & $3.92 \pm 0.56^{\mathrm{a}}$ & $4.65 \pm 0.33^{\mathrm{b}}$ \\
\hline
\end{tabular}

Data were expressed as means \pm standard deviations $(n=5)$. a,b Values with different superscripts in the same row differ significantly $(P<0.05)$.
The 16S rDNA sequences have been deposited in GenBank (SRA accession: PRJNA612427). A total of 1,627,645 reads were obtained from all samples with $53.13 \%(\mathrm{G}+\mathrm{C})$. Diets containing $B$. subtilis showed no effects in $\alpha$-diversity of ace, chao 1 , shannon, and simpson indices (Table 4). As shown in Figure 2, PCoA and PCA plots showed a good separation of cecal microbiota communities between two groups.

At family level, the main families were Ruminococcaceae, Lachnospiraceae, Lactobacillaceae, Clostridiales_vadinBB60_gro up, Erysipelotrichaceae, Defluviitaleaceae, o_Gastranaerophil ales_Unclassified, Enterobacteriaceae, Christensenellaceae, and Akkermansiaceae in chickens fed with $B$. subtilis. The relative abundance of Ruminococcaceae was increased after feeding with the B. subtilis diet (Figure 3A). At the genus level, the dominant species Faecalibacterium $(13.18 \%)$ and Lactobacillus (6.62\%) in chickens fed with the $B$. subtilis diet were significantly higher than that of controls (Figure 3B). Moreover, the relative abundance of Oscillibacter was increased after feeding B. subtilis, while the percentage of Lachnoclostridium, Butyricicoccus, and Flavonifractor showed a significant decrease (Figure 3C; $P<0.05$ ).

TABLE 4 | $\alpha$-diversity of microbial community structure.

\begin{tabular}{lcccc}
\hline Sample & Ace & Chao1 & Shannon & Simpson \\
\hline Bacillus subtilis & $143.06 \pm 9.23$ & $144.14 \pm 11.14$ & $5.26 \pm 0.32$ & $0.94 \pm 0.02$ \\
Control & $146.37 \pm 1.80$ & $149.83 \pm 8.17$ & $5.21 \pm 0.25$ & $0.95 \pm 0.00$
\end{tabular}

Data were expressed as means \pm standard deviations $(n=3)$. 

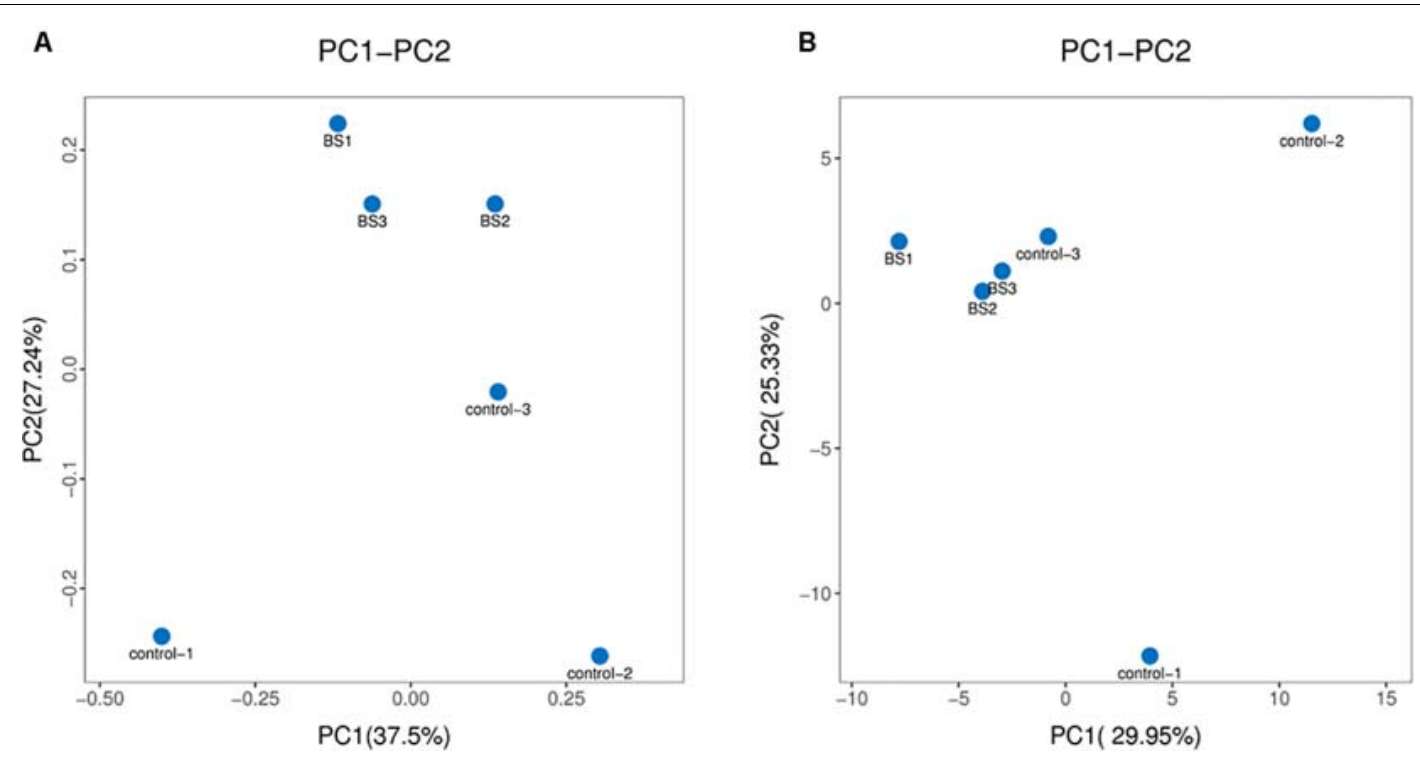

FIGURE 2 | $\beta$-diversity analysis of microbial communities. (A) Principal co-ordinates analysis (PCoA) plot based on Brary-Curtis distances $(n=3)$. (B) Principal component analysis (PCA) plot based on the distribution of bacterial community.

\section{Effect of Dietary B. subtilis on Cytokine Transcript Levels of the Spleen}

To investigate the role of $B$. subtilis in innate immunity, the transcript levels of immunity-related genes in the spleen were conducted by qPCR at 14 and 21 days. Our results showed that the major pro-inflammatory factors in the chickens were upregulated after feeding probiotics. Expressions of toll-like receptor 4 (Tlr4) and major histocompatibility complex $(M h c)$ $I I-\alpha$ were significantly upregulated by 4.35 - and 2.13-fold, respectively, at 14 days (Figures 4A,B). The signal transducing adaptor protein Myd88 was significantly upregulated by 8.90 - and 2.77-fold at 14 and 21 days, respectively (Figure 4C). Next, the expression of the downstream signaling molecule, nuclear factor kappa B $(N F-\kappa B)$ was also upregulated after feeding the diet with $B$. subtilis (Figure 4D). However, interferon (Ifn)- $\alpha$ expression showed no significant difference in chickens fed the B. subtilis diet compared to the controls (Figure 4E). At 14 and 21 days, the proinflammatory cytokines $I l 1 \beta$, Il6, and $I l 8$ showed obvious changes in chickens fed the B. subtilis (Figures $4 \mathrm{~F}-\mathbf{H}$ ).

\section{Survival Rate and E. coli Content}

As shown in Figure 5A, the survival rate of chickens fed with the B. subtilis diet was $66.67 \%$, which was significantly higher than in the controls. The chickens in the control group died at $10 \mathrm{~h}$ after challenge. The mortality rate of control group reached the highest at 2 days post-infection (dpi) and then decreased. At $5 \mathrm{dpi}$, the survival rate of chickens fed with $B$. subtilis was $16.67 \%$, and none of the chickens died after $6 \mathrm{dpi}$. However, the mortality rate of the probiotic group was $33.33 \%$, and none of the chickens died after 3 dpi. The chickens resumed the feed intake and drinking water at 3 dpi. Taken together, in the B. subtilis group, the time of death was delayed, and the number of deaths were fewer after E. coli infection.
E. coli contents in heart, liver, spleen, lung, and kidney were detected at 1 and 3 dpi. As shown in Figures 5B,C, the amount of $E$. coli in the test tissues of chickens fed with $B$. subtilis were lower than that of controls. Especially, E. coli contents in spleens and lungs of chickens fed with probiotics were $1.5 \times 10^{5} \mathrm{cfu} / \mathrm{g}$ and $6.1 \times 10^{4} \mathrm{cfu} / \mathrm{g}$, which were lower than $1.7 \times 10^{8} \mathrm{cfu} / \mathrm{g}$ and $3.3 \times 10^{7} \mathrm{cfu} / \mathrm{g}$ of controls, respectively $(P<0.05)$. At $3 \mathrm{dpi}$, the number of $E$. coli in tested tissues had declined compared with 1 dpi (Figure 5C).

\section{Expression of Innate Immune-Related Genes in the Spleen of the Infected Chickens}

We next studied the expression of innate immune-related genes induced by $B$. subtilis after $E$. coli infection. The induction of innate immunity-related genes were expressed more frequently in spleens at $1 \mathrm{dpi}$, and the up-regulation decreased at $3 \mathrm{dpi}$. Administration of B. subtilis significantly increased the Tlr4, Myd88, and NF- $\kappa B$ by 2-22-fold (Figures 6A,B,D, respectively). The expression of $M h c I I-\alpha$ was upregulated 79.31-fold on $1 \mathrm{dpi}$ (Figure 6C). The pro-inflammatory cytokines, Ifn- $\alpha$, Ill $\beta$, Il6, and $I l 8 \mathrm{mRNA}$ levels showed elevated expressions compared with control chickens (Figures 6E-H). In particular, the expression of Ill $\beta$ and $I l 6$ were upregulated by 24.33 - and 74.40 -fold at $1 \mathrm{dpi}$, respectively. At $3 \mathrm{dpi}$, the expressions of Il1 $\beta$ and Il6 were up-regulated by 3.80- and 2.31-fold, respectively (Figures 6F,G).

\section{DISCUSSION}

Increasing evidence supports the concept that dietary B. subtilis can promote the growth performance of animals (Lee S.H. et al., 2014; Musa et al., 2019; Sokale et al., 2019). However, some other 

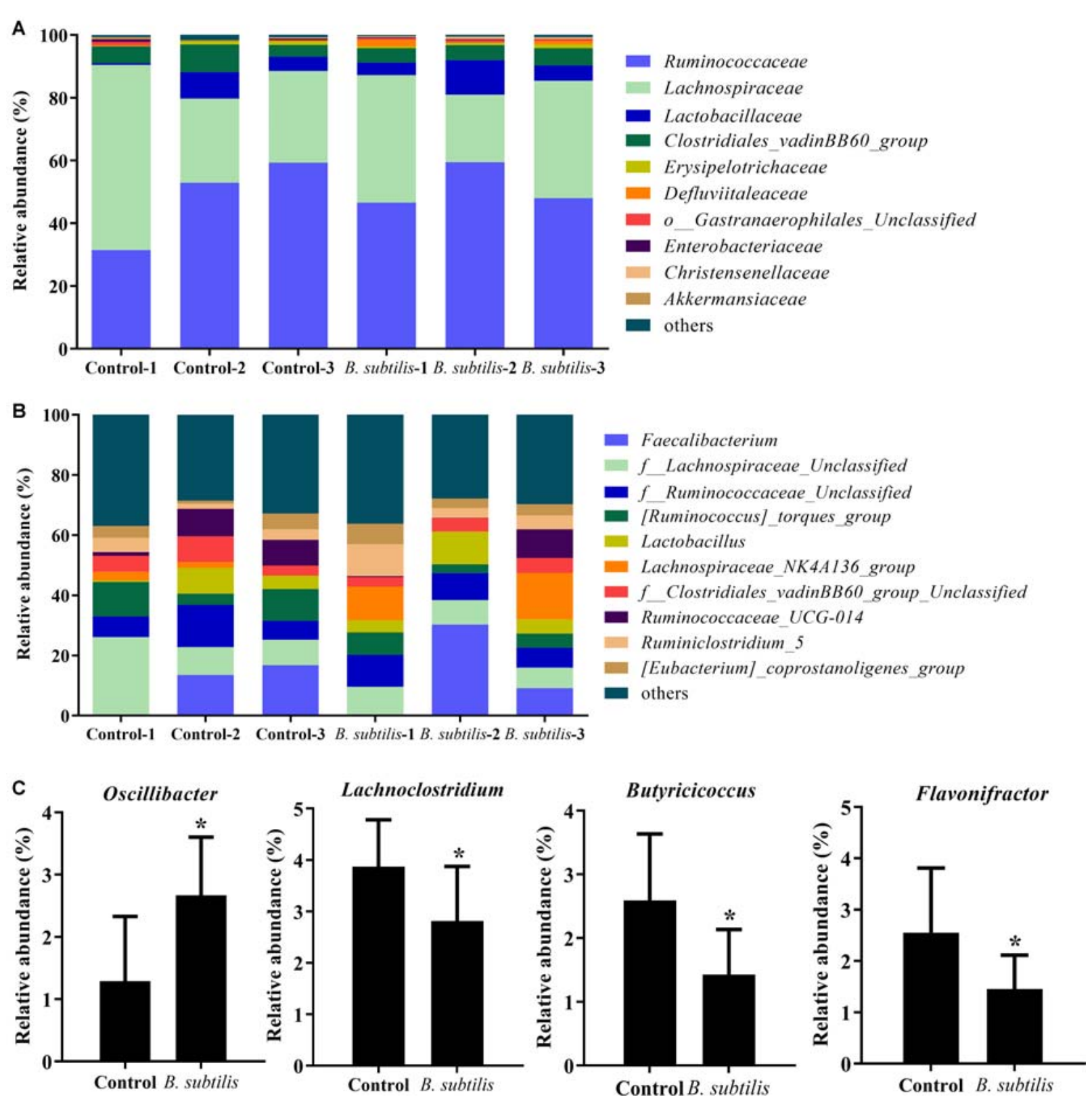

FIGURE 3 | Effects of dietary Bacillus subtilis on cecal microbes at different taxonomic levels in chickens. (A) Relative abundance of the top 10 bacteria at the family level. (B) Relative abundance of the top 10 bacteria at the genus level. (C) Relative abundance of genera Oscillibacter, Lactobacillus, Butyricicoccus, and Faecalibacterium. Bars were expressed as means \pm standard $(n=3)$. ${ }^{\star} P<0.05$.

studies showed that there is no relationship of B. subtilis with average daily gain and feed conversion ratio (Lee K.W. et al., 2014; Zhang et al., 2017). It has been demonstrated that probiotic functions are affected by environmental temperature, probiotic strains, administration levels, and animal species. In this study, we investigated growth performance, serum immunoglobulins, intestinal homeostasis, immune responses, and disease resistance of chickens with B. subtilis strains BG5 and BYS2 addition.

In this study, administration of $B$. subtilis into diets caused an increase in chicken body weight gain. Higher digestive and absorption of nutrients in animals fed with probiotics might be due to improved intestinal morphology, modulated gut environment, and stimulated immune responses (Choi et al., 2011). The changes of intestinal morphology including the increase in $\mathrm{VH}$ and $\mathrm{VH} / \mathrm{CD}$ ratio indicated an improved absorptive surface area and digestive and absorption capacities (Sen et al., 2012). Crypts are the sites of proliferation and differentiation of intestinal epithelial cells that promote villus growth. Similarly with the previous study the previous study (Ma et al., 2018), our results showed that chickens fed with 

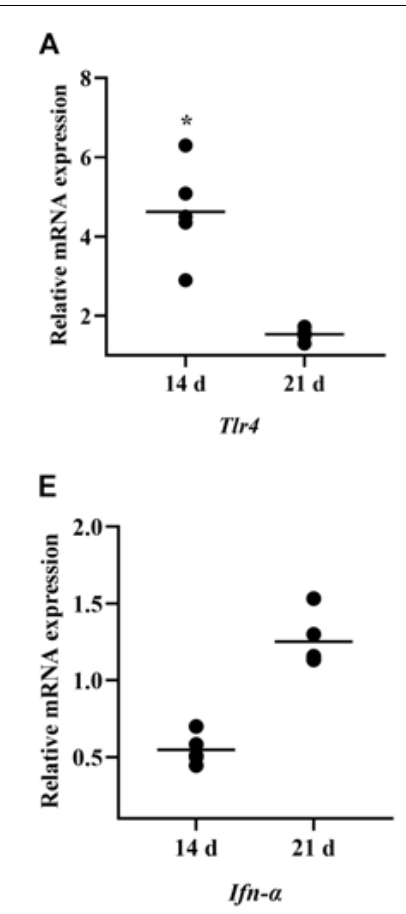

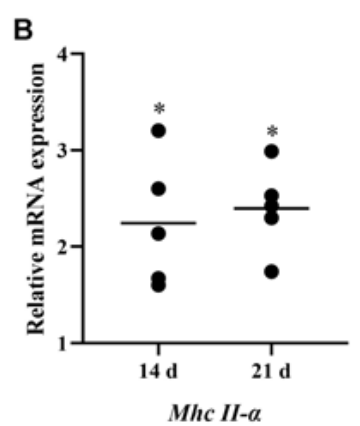

$\mathbf{F}$

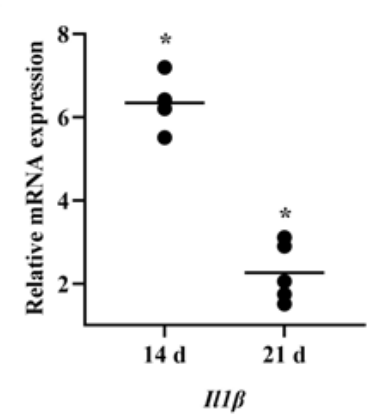

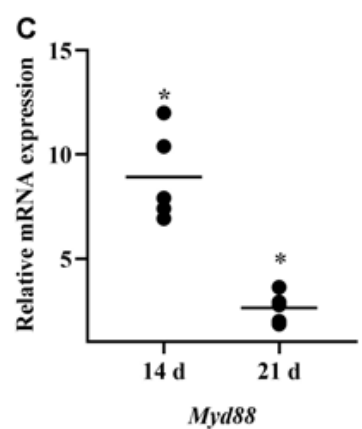

G

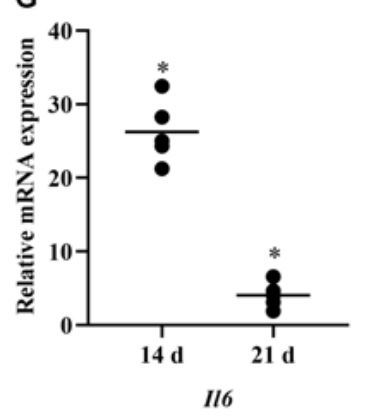

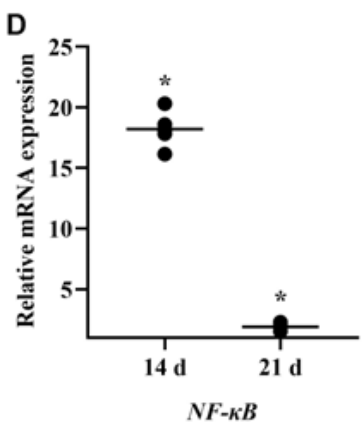

H

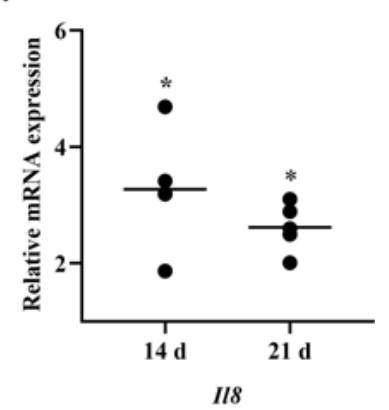

FIGURE 4 | Effects of dietary Bacillus subtilis on the levels of transcripts of immune-related genes. (A) Tir4, (B) Mhc II- $\alpha$, (C) Myd88, (D) NF-kB, (E) Ifn- $\alpha$, (F) $/ 11 \beta$, (G) $/ / 6$, (H) //8. The relative mRNA expression represent the target gene expressions of diets with $B$. subtilis vs. controls. Bars were expressed as geometric mean $(n=5) .{ }^{*} P<0.05$.
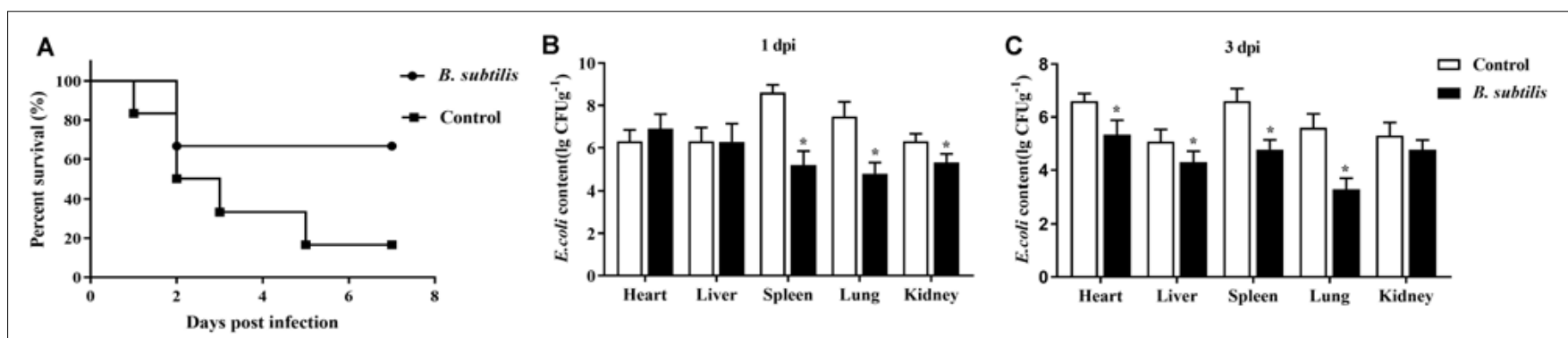

FIGURE 5 | Effects of dietary Bacillus subtilis on disease resistance against Escherichia coli. (A) The survival rate of chickens after infection with E. coli ( $n=10)$. (B,C) E. coli contents of infected chickens at 1 and 3 dpi, respectively $\left(\log _{10} \mathrm{CFU} \mathrm{g}{ }^{-1}\right)$. Bars were expressed as means \pm standard $(n=5)$. ${ }^{*} P<0.05$.

B. subtilis had greater $\mathrm{VH}$, shallower $\mathrm{CD}$, and higher $\mathrm{VH} / \mathrm{CD}$ ratio in the jejunum, which might also explain the elevated growth performance in chickens. Furthermore, growth performance improvement is related to nutrients and extracellular digestive enzymes produced by B. subtilis (Sahu et al., 2008). In our previous research, we have proved that $B$. subtilis used in this study had good performance in protease activities.

Serum IgA and IgM increases found in chickens fed with B. subtilis diets in the present study reflected an improvement in immune function. These results are in agreement with previous research in which administration of $B$. subtilis promoted the increase in IgA concentrations in pigs (Lee S.H. et al., 2014). The gastrointestinal tract is particularly sensitive to stress and especially can cause changes in normal intestinal microbiota. It is widely believed that the main reason probiotic-related growth improvement performance and intestinal morphology is intestinal microbiota modulation (Crisol-Martínez et al., 2017). In the present study, administration of $B$. subtilis modified the relative abundance of cecal microbiota. In particular, the relative abundance of Ruminococcaceae, Faecalibacterium, Lactobacillus, and Oscillibacter were increased after feeding a B. subtilis diet. The Ruminococcaceae species are positively related to body weight, and they play a vital role in fiber degradation and butyrate production (Zhao et al., 2017; Fang et al., 2019). F. prausnitzii is a functionally important commensal bacterium, which has an impact on the physiology and health of host. The amount of $F$. prausnitzii is negatively associated with inflammatory bowel disease and colorectal cancer. F. prausnitzii is the only species identified in the genus Faecalibacterium (Benevides et al., 2017; Ferreira-Halder et al., 2017). As 

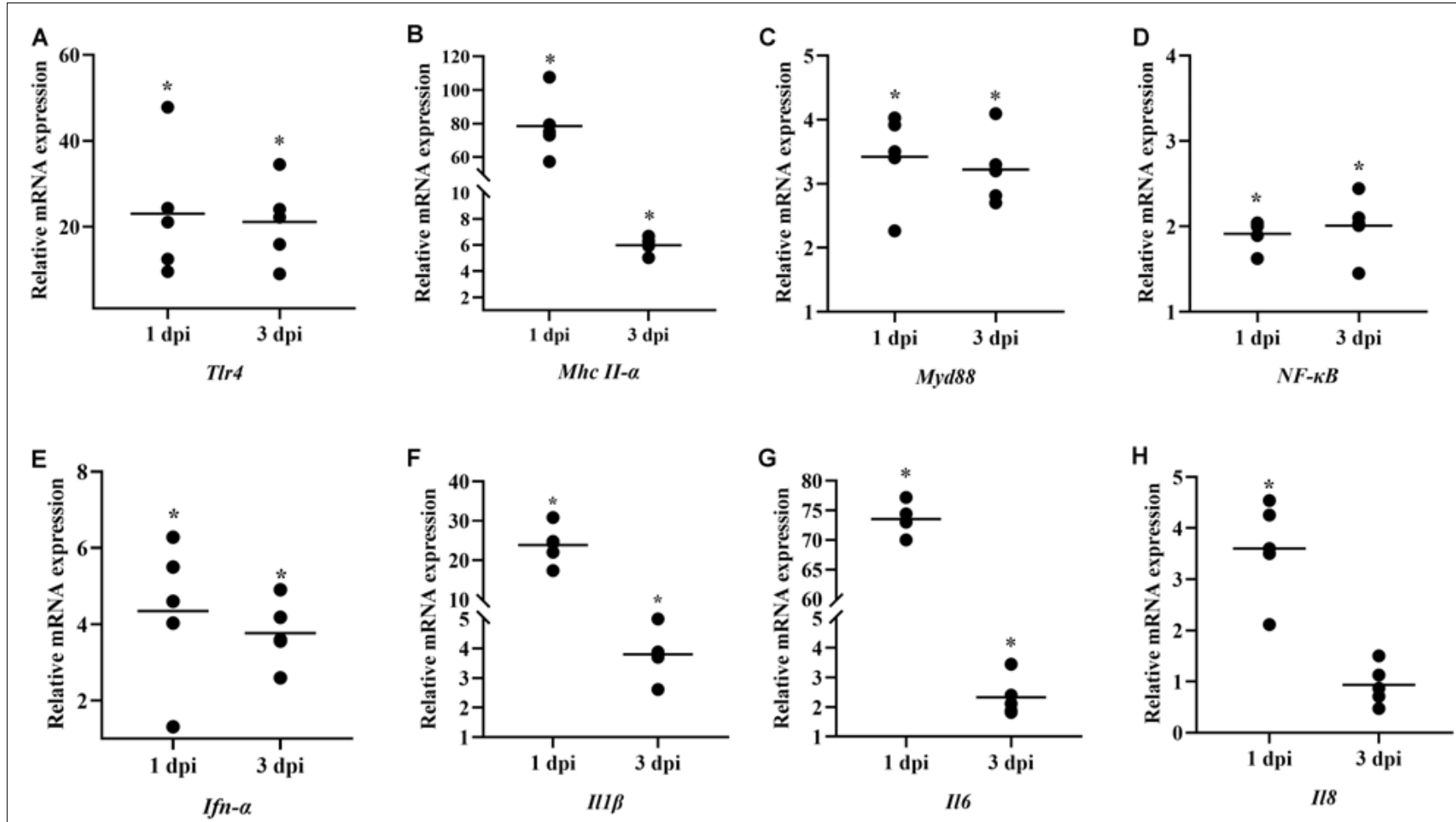

FIGURE 6 | Effects of dietary Bacillus subtilis on transcript levels of immune-related genes after infection with E. coli. (A) T/r4, (B) Mhc II- $\alpha$, (C) Myd88, (D) NF-кB, (E) Ifn- $\alpha, \mathbf{( F )}\|1 \beta,(\mathbf{G})\| 6,(\mathbf{H}) \| / 8$. The relative mRNA expression represents the target gene expression of the diet containing $B$. subtilis rather than that of the controls after infection with $E$. coli. Bars were expressed as geometric mean $(n=5) .{ }^{*} P<0.05$.

probiotics, Lactobacillus and B. subtilis have been suggested as the greatest potential therapy. Oscillibacter is a newly discovered genus that plays a crucial role in maintaining mucosal homeostasis and anti-inflammatory functions (Lopetuso et al., 2013; Wang et al., 2018). In contrast, Flavonifractor was reported to induce oxidative stress and inflammation (Coello et al., 2019). Lachnoclostridium and Butyricicoccus are related to chronic low-grade inflammation and metabolic diseases and negatively related to body weight in human and animals (Guo et al., 2018; Fang et al., 2019; Kong et al., 2019). Hence, the relevant increase in Ruminococcaceae and Lactobacillus and decrease in Lachnoclostridium and Butyricicoccus could partially explain the increase in body weights in chickens fed with B. subtilis. Taken together, our results indicate that diets with $B$. subtilis can improve growth performance, modulate intestinal microbiota, and maintain intestinal homeostasis.

It is generally believed that probiotics exert opposite effects on pathogenic bacteria, including inhibition rather than stimulation of proinflammatory responses (Jijon et al., 2004). However, several studies emphasized the importance of relating "physiological inflammation" that is caused by symbiotic microbiota immune system development and the response to intestinal pathogens. Accordingly, the pro-inflammatory response induced by probiotics is currently under exploration. TLRs, as a type of pattern recognition receptor, can activate immune responses and regulate inflammatory responses. In the previous study, oral administration of probiotics caused an increase in Tlr3 and Tlr4 gene expressions (Cario and Podolsky, 2000). Moreover, changes in the intestinal microbiota due to the $B$. subtilis addition may have also led to the different activation of pattern recognition receptors. Our results showed that after dietary administration of $B$. subtilis increased the mRNA expression level of Tlr4. Myd88 is a kind of adapter protein that plays an effective role in activating the downstream $N F-\kappa B$ signaling pathway. In the present study, application of $B$. subtilis significantly increased the expression of $M y d 88$ and $N F-\kappa B$. Similarly, diets with Saccharomyces boulardii and $B$. subtilis activated the innate immune response that was dependent on the Myd88 signaling pathway (Rajput et al., 2017). $N F-\kappa B$ plays a beneficial role in protecting the development of systemic inflammation (Chen et al., 2003). In this study, dietary with B. subtilis significantly increased the mRNA expression levels of proinflammatory cytokines (Il1 $\beta, I l 6$, and Il8). Our results were similar as those previous study in which the probiotic VSL\#3 activated Tlr9mediated activation of $N F-\kappa B$ and promoted proinflammatory cytokines Il6 and Il12 (Rachmilewitz et al., 2004). Taken together, diets containing B. subtilis could trigger Tlr4 and activate proinflammatory cytokine production to develop innate immunity responses in chickens.

Probiotics can help animals resist pathogenic bacteria infections. In a previous study, B. subtilis DSM 32315 significantly 
enhanced the disease resistance after a necrotic enteritis challenge (Whelan et al., 2019). In the current study, the survival rate of chickens fed with $B$. subtilis was higher than that of controls after infected with E. coli. The E. coli contents in the hearts, livers, spleens, lungs, and kidneys of chickens fed with B. subtilis were lower than that of controls. This conclusion was supported by growth performance promotion, increases in serum immunoglobulins, promotion of intestinal homeostasis, and enhances immune responses. It is worth noting that the major pro-inflammatory factors (Ifn- $\alpha, I l 1 \beta, I l 6$, and Ils) were upregulated in the $B$. subtilis feeding group after being challenged with E. coli. Ill $\beta$ is the major proinflammatory cytokine, which induces the production of itself, other proinflammatory cytokines and chemokines (such as $I l 6, I l 8$, and Tnf- $a$ ). In return, they can activate inflammation and recruit neutrophils and macrophages which can phagocytize and kill bacteria. Previous study showed that probiotics administration almost complete prevent mucosal damage, with preservation of the normal villi morphology and minimal inflammatory infiltration. The results were related to activation of NF- $\kappa \mathrm{B}$ and production of TNF- $\alpha$, coupled with restitution of normal intestinal epithelial barrier function (Pagnini et al., 2010). In fact, activation of NF- $\kappa$ B plays a beneficial role in epithelial cells. Activation of NF- $\kappa$ B has a similar protective effect on the development of systemic inflammation, but not for local injury (Chen et al., 2003). The proinflammatory cytokine, is fundamental in establishing the inflammatory disease can have an opposing, protective effect during disease initiation. Because proinflammatory cytokines (such as IFN- $\gamma$, IL-6, and TNF- $\alpha$ ) are part of the innate immune system, one hypothesis is that proinflammatory cytokines are partly directed against luminal antigens (bacterial or food antigens) that would otherwise threaten mucosal integrity. We deduced that $B$. subtilis prevent bacterial disease by mechanisms involving activation of $N F-\kappa B$, and maintain the integrity of the intestinal epithelium.

\section{CONCLUSION}

In conclusion, diets containing B. subtilis appear to improve growth performance, serum immunoglobulin levels, intestinal homeostasis, immune responses, and disease resistance in chickens. In addition, B. subtilis can trigger Tlr4 and activate proinflammatory responses in chickens. Our results indicated that $B$. subtilis may be an antibiotic alternative approach for controlling bacterial infection.

\section{REFERENCES}

Abdelqader, A., Al-Fataftah, A.-R., and Daş, G. (2013). Effects of dietary Bacillus subtilis and inulin supplementation on performance, eggshell quality, intestinal morphology and microflora composition of laying hens in the late phase of production. Anim. Feed Sci. Technol. 179, 103-111. doi: 10.1016/j.anifeedsci. 2012.11.003

Abudabos, A. M., Alhouri, H. A., Alhidary, I. A., Nassan, M. A., and Swelum, A. A. (2019). Ameliorative effect of Bacillus subtilis, Saccharomyces boulardii, oregano, and calcium montmorillonite on growth, intestinal histology, and

\section{DATA AVAILABILITY STATEMENT}

The original contributions presented in the study are publicly available. This data can be found here: https://www.ncbi.nlm.nih. gov/sra/?term=PRJNA612427.

\section{ETHICS STATEMENT}

The animal study was reviewed and approved by the Committee on the Ethics of Animal Experiments of Yangzhou University.

\section{AUTHOR CONTRIBUTIONS}

MG and ML designed and conducted the study, performed most of the experiments, and wrote the manuscript. CZ performed the calculation with support from XZ. YW discussed the results and revised the manuscript. All authors contributed to the article and approved the submitted version.

\section{FUNDING}

This work was supported by the China Postdoctoral Science Foundation (2019M651987), the National Key Research and Development Project of China (2016YFD0501609), the National Natural Science Foundation of China (31902236), the Natural Science Foundation of the Jiangsu Higher Education Institutions of China (19KJB230002), and the Project Funded by the Priority Academic Program Development of Jiangsu Higher Education Institutions (PAPD).

\section{SUPPLEMENTARY MATERIAL}

The Supplementary Material for this article can be found online at: https://www.frontiersin.org/articles/10.3389/fmicb. 2020.01768/full\#supplementary-material

FIGURE S1 | Effects of dietary Bacillus subtilis on intestinal morphology of chickens. The representative photomicrographs of hematoxylin and eosin (H\&E)-stained sections of intestinal sections: (A) Duodenum, (B) Jejunum, and (C) lleum in chickens fed with $B$. subtilis. (D-F) Show the duodenum, jejunum, and ileum of chickens fed with the control diet, respectively, at $40 \times$ original magnification.

blood metabolites on Salmonella-infected broiler chicken. Environ. Sci. Pollut. Res. 26, 16274-16278. doi: 10.1007/s11356-019-05105-1

Al-Fataftah, A.-R., and Abdelqader, A. (2014). Effects of dietary Bacillus subtilis on heat-stressed broilers performance, intestinal morphology and microflora composition. Anim. Feed Sci. Technol. 198, 279-285. doi: 10.1016/j.anifeedsci. 2014.10.012

Benevides, L., Burman, S., Martin, R., Robert, V., Thomas, M., Miquel, S., et al. (2017). New insights into the diversity of the genus Faecalibacterium. Front. Microbiol. 8:1790. doi: 10.3389/fmicb.2017. 01790 
Cario, E., and Podolsky, D. K. (2000). Differential alteration in intestinal epithelial cell expression of toll-like receptor 3 (TLR3) and TLR4 in inflammatory bowel disease. Infect. Immun. 68, 7010-7017. doi: 10.1128/iai.68.12.7010-7017.2000

Chen, L.-W., Egan, L., Li, Z.-W., Greten, F. R., Kagnoff, M. F., and Karin, M. (2003). The two faces of IKK and NF- $\mathrm{B}$ inhibition: prevention of systemic inflammation but increased local injury following intestinal ischemiareperfusion. Nat. Med. 9, 575-581. doi: 10.1038/nm849

Choi, J. Y., Shinde, P. L., Ingale, S. L., Kim, J. S., Kim, Y. W., Kim, K. H., et al. (2011). Evaluation of multi-microbe probiotics prepared by submerged liquid or solid substrate fermentation and antibiotics in weaning pigs. Livest. Sci. 138, 144-151. doi: 10.1016/j.livsci.2010.12.015

Coello, K., Hansen, T. H., Sørensen, N., Munkholm, K., Kessing, L. V., Pedersen, O., et al. (2019). Gut microbiota composition in patients with newly diagnosed bipolar disorder and their unaffected first-degree relatives. Brain Behav. Immun. 75, 112-118. doi: 10.1016/j.bbi.2018.09.026

Crisol-Martínez, E., Stanley, D., Geier, M. S., Hughes, R. J., and Moore, R. J. (2017). Understanding the mechanisms of zinc bacitracin and avilamycin on animal production: linking gut microbiota and growth performance in chickens. Appl. Microbiol. Biotechnol. 101, 4547-4559. doi: 10.1007/s00253-0178193-9

Dowarah, R., Verma, A. K., Agarwal, N., Singh, P., and Singh, B. R. (2018). Selection and characterization of probiotic lactic acid bacteria and its impact on growth, nutrient digestibility, health and antioxidant status in weaned piglets. PLoS One 13:e0192978. doi: 10.1371/journal.pone.019 2978

EFSA (2007). Introduction of a Qualified Presumption of Safety (QPS) approach for assessment of selected microorganisms referred to EFSA-opinion of the scientific committee. EFSA J. 5:587. doi: 10.2903/j.efsa.2007.587

Elshaghabee, F. M., Rokana, N., Gulhane, R. D., Sharma, C., and Panwar, H. (2017). Bacillus as potential probiotics: status, concerns, and future perspectives. Front. Microbiol. 8:1490. doi: 10.3389/fmicb.2017.01490

Fang, S., Chen, X., Zhou, L., Wang, C., Chen, Q., Lin, R., et al. (2019). Faecal microbiota and functional capacity associated with weaning weight in meat rabbits. Microb. Biotechnol. 12, 1441-1452. doi: 10.1111/1751-7915.13485

Ferreira-Halder, C. V., De Sousa Faria, A. V., and Andrade, S. S. (2017). Action and function of Faecalibacterium prausnitzii in health and disease. Best Pract. Res. Clin. Gastroenterol. 31, 643-648. doi: 10.1016/j.bpg.2017.09.011

Fuller, R. (1989). Probiotics in man and animals. J. Appl. Bacteriol. 66, 365-378. doi: 10.1111/j.1365-2672.1989.tb05105.x

Gadde, U. D., Oh, S., Lee, Y., Davis, E., Zimmerman, N., Rehberger, T., et al. (2017). Dietary Bacillus subtilis-based direct-fed microbials alleviate LPSinduced intestinal immunological stress and improve intestinal barrier gene expression in commercial broiler chickens. Res. Vet. Sci. 114, 236-243. doi: 10.1016/j.rvsc.2017.05.004

Gebert, S. (2007). Effect of a Bacillus-based direct-fed microbial on turkey poult performance and changes within the gastrointestinal microflora. Poult. Sci. 86:249. doi: 10.1016/j.livsci.2007.01.063

Guo, M., Wu, F., Hao, G., Qi, Q., Li, R., Li, N., et al. (2017). Bacillus subtilis improves immunity and disease resistance in rabbits. Front. Immunol. 8:354. doi: 10.3389/fmicb.2017.0354

Guo, Y., Huang, Z.-P., Liu, C.-Q., Qi, L., Sheng, Y., and Zou, D.-J. (2018). Modulation of the gut microbiome: a systematic review of the effect of bariatric surgery. Eur. J. Endocrinol. 178, 43-56. doi: 10.1530/eje-17-0403

Hong, H. A., Duc, L. H., and Cutting, S. M. (2005). The use of bacterial spore formers as probiotics. FEMS Microbiol. Rev. 29, 813-835. doi: 10.1016/j.femsre. 2004.12.001

Jijon, H., Backer, J., Diaz, H., Yeung, H., Thiel, D., Mckaigney, C., et al. (2004). DNA from probiotic bacteria modulates murine and human epithelial and immune function. Gastroenterology 126, 1358-1373. doi: 10.1053/j.gastro.2004. 02.003

Knap, I., Kehlet, A., Bennedsen, M., Mathis, G., Hofacre, C., Lumpkins, B., et al. (2011). Bacillus subtilis (DSM17299) significantly reduces Salmonella in broilers. Poult. Sci. 90, 1690-1694. doi: 10.3382/ps.2010-01056

Kong, C., Gao, R., Yan, X., Huang, L., and Qin, H. (2019). Probiotics improve gut microbiota dysbiosis in obese mice fed a high-fat or high-sucrose diet. Nutrition 60, 175-184. doi: 10.1016/j.nut.2018.10.002

Larsen, N., Thorsen, L., Kpikpi, E. N., Stuer-Lauridsen, B., Cantor, M. D., Nielsen, B., et al. (2014). Characterization of Bacillus spp. strains for use as probiotic additives in pig feed. Appl. Microbiol. Biotechnol. 98, 1105-1118. doi: 10.1007/ s00253-013-5343-6

Lee, K., Lillehoj, H. S., and Siragusa, G. R. (2010). Direct-fed microbials and their impact on the intestinal microflora and immune system of chickens. J. Poult. Sci. 47, 106-114. doi: 10.2141/jpsa.009096

Lee, K.-W., Kim, D. K., Lillehoj, H. S., Jang, S. I., and Lee, S.-H. (2015). Immune modulation by Bacillus subtilis-based direct-fed microbials in commercial broiler chickens. Anim. Feed Sci. Technol. 200, 76-85. doi: 10.1016/j.anifeedsci. 2014.12.006

Lee, K.-W., Lillehoj, H. S., Jang, S. I., and Lee, S.-H. (2014). Effects of salinomycin and Bacillus subtilis on growth performance and immune responses in broiler chickens. Res. Vet. Sci. 97, 304-308. doi: 10.1016/j.rvsc.2014. 07.021

Lee, S. H., Ingale, S. L., Kim, J. S., Kim, K. H., Lokhande, A., Kim, E. K., et al. (2014). Effects of dietary supplementation with Bacillus subtilis LS 1-2 fermentation biomass on growth performance, nutrient digestibility, cecal microbiota and intestinal morphology of weanling pig. Anim. Feed Sci. Technol. 188, 102-110. doi: 10.1016/j.anifeedsci.2013.12.001

Lopetuso, L. R., Scaldaferri, F., Petito, V., and Gasbarrini, A. (2013). Commensal clostridia: leading players in the maintenance of gut homeostasis. Gut Pathog. 5:23. doi: 10.1186/1757-4749-5-23

Lupindu, A. M., Dalsgaard, A., Msoffe, P. L., Ngowi, H. A., Mtambo, M. M., and Olsen, J. E. (2015). Transmission of antibiotic-resistant Escherichia coli between cattle, humans and the environment in peri-urban livestock keeping communities in Morogoro, Tanzania. Prevent. Vet. Med. 118, 477-482. doi: 10.1016/j.prevetmed.2014.12.005

Ma, Y., Wang, W., Zhang, H., Wang, J., Zhang, W., Gao, J., et al. (2018). Supplemental Bacillus subtilis DSM 32315 manipulates intestinal structure and microbial composition in broiler chickens. Sci. Rep. 8, 1-13.

Musa, B. B., Duan, Y., Khawar, H., Sun, Q., Ren, Z., Elsiddig Mohamed, M. A., et al. (2019). Bacillus subtilis B21 and Bacillus licheniformis B26 improve intestinal health and performance of broiler chickens with clostridium perfringensinduced necrotic enteritis. J. Anim. Physiol. Anim. Nutr. 103, 1039-1049.

Ng, S. C., Hart, A. L., Kamm, M. A., Stagg, A. J., and Knight, S. C. (2009). Mechanisms of action of probiotics: recent advances. Inflamm. Bowel Dis. 15, 300-310. doi: 10.1002/ibd.20602

Pagnini, C., Saeed, R., Bamias, G., Arseneau, K. O., Pizarro, T. T., and Cominelli, F. (2010). Probiotics promote gut health through stimulation of epithelial innate immunity. Proc. Natl. Acad. Sci. U.S.A. 107, 454-459. doi: 10.1073/pnas. 0910307107

Rachmilewitz, D., Katakura, K., Karmeli, F., Hayashi, T., Reinus, C., Rudensky, B., et al. (2004). Toll-like receptor 9 signaling mediates the anti-inflammatory effects of probiotics in murine experimental colitis. Gastroenterology 126, 520528. doi: 10.1053/j.gastro.2003.11.019

Rajput, I. R., Hussain, A., Li, Y. L., Zhang, X., Xu, X., Long, M. Y., et al. (2014). Saccharomyces boulardii and Bacillus subtilis B10 modulate TLRs mediated signaling to induce immunity by chicken BMDCs. J. Cell. Biochem. 115, 189198. doi: $10.1002 /$ jcb. 24650

Rajput, I. R., Ying, H., Yajing, S., Arain, M. A., Weifen, L., Ping, L., et al. (2017). Saccharomyces boulardii and Bacillus subtilis B10 modulate TLRs and cytokines expression patterns in jejunum and ileum of broilers. PLoS One 12:e0173917. doi: 10.1371/journal.pone.0173917

Sahu, M. K., Swarnakumar, N. S., Sivakumar, K., Thangaradjou, T., and Kannan, L. (2008). Probiotics in aquaculture: importance and future perspectives. Indian J. Microbiol. 48, 299-308. doi: 10.1007/s12088-008-0024-3

Schouler, C., Schaeffer, B., Brée, A., Mora, A., Dahbi, G., Biet, F., et al. (2012). Diagnostic strategy for identifying avian pathogenic Escherichia coli based on four patterns of virulence genes. J. Clin. Microbiol. 50, 1673-1678. doi: 10.1128/ jcm.05057-11

Sen, S., Ingale, S. L., Kim, Y. W., Kim, J. S., Kim, K. H., Lohakare, J. D., et al. (2012). Effect of supplementation of Bacillus subtilis LS 1-2 to broiler diets on growth performance, nutrient retention, caecal microbiology and small intestinal morphology. Res. Vet. Sci. 93, 264-268. doi: 10.1016/j.rvsc.2011. 05.021

Sokale, A., Menconi, A., Mathis, G., Lumpkins, B., Sims, M., Whelan, R., et al. (2019). Effect of Bacillus subtilis DSM 32315 on the intestinal structural integrity and growth performance of broiler chickens under necrotic enteritis challenge. Poult. Sci. 98, 5392-5400. doi: 10.3382/ps/pez368 
Wang, C.-S.-E., Li, W.-B., Wang, H.-Y., Ma, Y.-M., Zhao, X.-H., Yang, H., et al. (2018). VSL\# 3 can prevent ulcerative colitis-associated carcinogenesis in mice. World J. Gastroenterol. 24:4254.

Whelan, R. A., Doranalli, K., Rinttilä, T., Vienola, K., Jurgens, G., and Apajalahti, J. (2019). The impact of Bacillus subtilis DSM 32315 on the pathology, performance, and intestinal microbiome of broiler chickens in a necrotic enteritis challenge. Poult. Sci. 98, 3450-3463. doi: 10.3382/ps/pe y500

Zhang, L., Bai, K., Zhang, J., Xu, W., Huang, Q., and Wang, T. (2017). Dietary effects of Bacillus subtilis fmbj on the antioxidant capacity of broilers at an early age. Poult. Sci. 96, 3564-3573. doi: 10.3382/ps/pex172

Zhao, L., Zhang, Q., Ma, W., Tian, F., Shen, H., and Zhou, M. (2017). A combination of quercetin and resveratrol reduces obesity in high-fat diet-fed rats by modulation of gut microbiota. Food Funct. 8, 4644-4656. doi: 10.1039/ c7fo0 1383 c

Conflict of Interest: The authors declare that the research was conducted in the absence of any commercial or financial relationships that could be construed as a potential conflict of interest.

Copyright (c) $2020 \mathrm{Guo}$, Li, Zhang, Zhang and Wu. This is an open-access article distributed under the terms of the Creative Commons Attribution License (CC BY). The use, distribution or reproduction in other forums is permitted, provided the original author(s) and the copyright owner(s) are credited and that the original publication in this journal is cited, in accordance with accepted academic practice. No use, distribution or reproduction is permitted which does not comply with these terms. 